\title{
Glutathione depletion in human erythrocytes and rat liver: a study on the interplay between bioactivation and inactivation functions of liver and blood
}

Citation for published version (APA):

Palmen, N. G. M., \& Evelo, C. T. A. (1996). Glutathione depletion in human erythrocytes and rat liver: a study on the interplay between bioactivation and inactivation functions of liver and blood. Toxicology in Vitro, 10, 273-281. https://doi.org/10.1016/0887-2333(96)00002-1

Document status and date:

Published: 01/01/1996

DOI:

10.1016/0887-2333(96)00002-1

Document Version:

Publisher's PDF, also known as Version of record

\section{Document license:}

Taverne

Please check the document version of this publication:

- A submitted manuscript is the version of the article upon submission and before peer-review. There can be important differences between the submitted version and the official published version of record.

People interested in the research are advised to contact the author for the final version of the publication, or visit the DOI to the publisher's website.

- The final author version and the galley proof are versions of the publication after peer review.

- The final published version features the final layout of the paper including the volume, issue and page numbers.

Link to publication

\footnotetext{
General rights rights.

- You may freely distribute the URL identifying the publication in the public portal. please follow below link for the End User Agreement:

www.umlib.nl/taverne-license

Take down policy

If you believe that this document breaches copyright please contact us at:

repository@maastrichtuniversity.nl

providing details and we will investigate your claim.
}

Copyright and moral rights for the publications made accessible in the public portal are retained by the authors and/or other copyright owners and it is a condition of accessing publications that users recognise and abide by the legal requirements associated with these

- Users may download and print one copy of any publication from the public portal for the purpose of private study or research.

- You may not further distribute the material or use it for any profit-making activity or commercial gain

If the publication is distributed under the terms of Article $25 \mathrm{fa}$ of the Dutch Copyright Act, indicated by the "Taverne" license above, 


\title{
Glutathione Depletion in Human Erythrocytes and Rat Liver: a Study on the Interplay Between Bioactivation and Inactivation Functions of Liver and Blood
}

\author{
N. G. M. PALMEN† and C. T. A. EVELO* \\ Department of Pharmacology, Toxicology Section, University of Limburg, PO Box 616 ,
} 6200 MD Maastricht, The Netherlands

(Accepted 17 October 1995)

\begin{abstract}
The interplay between bioactivation and inactivation functions of human erythrocytes and rat liver was studied. Glutathione depletion was used as a measurc of the amount of reduced glutathione (GSH)-reactive compound. Iodoacetamide (IACA), N-ethylmaleimide (NEM) and diethyl maleate (DEM), which are electrophiles that need no metabolic activation, were able to deplete GSH in incubations with either aqueous GSH solution or erythrocytes. These results indicate that these compounds can pass the erythrocyte membrane. Cyclophosphamide (CP), 3-hydroxyacetanilide (3-HAA) and 2-methylfurane (2-MF) needed metabolic activation by rat liver microsomes to deplete glutathione in incubations with aqueous GSH solution or erythrocytes. By measuring the sum of both reduced and oxidized glutathione [ = total glutathione (GT)] it became clear that GSH-reactive metabolites are generated out of CP, 3-HAA and 2-MF by the action of microsomes and that these metabolites can pass through the erythrocyte membrane. As GT depletion was higher when microsomes of phenobarbital-pretreated rats were used, the metabolites were (are expected to be) generated by phenobarbital-inducible enzymes. GT was also depleted in incubations with haemolysate and 3-HAA or 2-MF but not in incubations with aqueous GSH solution, which indicates that erythrocyte cytosol can metabolize 3-HAA and 2-MF into GSH-reactive compounds. The pesticides monuron and monulinuron did not affect GT concentrations when aqueous GSH solution, haemolysate or erythrocytes with or without microsomal activating system were tested. When hepatocytes were incubated with 3-HAA or CP ( $2 \mathrm{~mm}$ ), about $2 \mathrm{~mm}$ of internal GT concentration was depleted. The hepatocytes excreted GSH-reactive metabolites generated from 3-HAA and CP (about $20 \%$ of the metabolites formed for 3-HAA). Erythrocyte GT was not depleted in co-incubations of hepatocytes and erythrocytes with 3-HAA. This can be explained by the amounts of GSH-reactive metabolites excreted by the hepatocytes, which would require very effective uptake by the erythrocytes in order to be detectable. Copyright (C) 1996 Elsevier Science Ltd
\end{abstract}

\section{INTRODUCTION}

Glutathione in its reduced form (GSH) and glutathione $S$-transferase (GST) have key roles in the detoxification of electrophilic compounds (DeLeve and Kaplowitz, 1991; Mannervik and Danielson, 1988; Meister and Anderson, 1983). Human erythrocytes contain a high concentration of GSH (about $2 \mathrm{mM}$ ) (Fazi et al., 1991) and a relatively high GST activity (1-6 U haemoglobin/g towards the common

*Author for correspondence

†Current address: Arbodienst Limburg, Maastricht, The Netherlands.

Abbreviations: $\quad \mathrm{CP}=$ cyclophosphamide; $\quad \mathrm{DEM}=$ diethyl maleate; $\mathbf{G S H}=$ reduced glutathione; $\mathrm{GST}=$ glutathione $S$-transferase; GT $=$ total glutathione; 3 -HAA $=$ 3-hydroxyacetanilide; IAcA = iodoacetamide; $\mathbf{L D H}=$ lactate dehydrogenase; MAS = microsomal activating system; 2-MF = 2-methylfurane; $\mathrm{MFO}=$ mixed-function oxidase; $\quad M L N=$ monulinuron; $\quad \mathrm{NEM}=N$-ethylmaleimide; $\mathrm{PB}=$ phenobarbital; PBS $=$ phosphate buffered saline; $\mathrm{TCA}=$ trichloracetic acid. substrate 1-chloro-2,4-dinitrobenzene) (Evelo and Henderson, 1992; Strange et al., 1982). Erythrocytes may therefore act as circulating bioreactors, conjugating electrophiles near or in the tissues where they originate. This may lead to consumption of erythrocyte GSH, and, in fact, various electrophiles are known to cause glutathione depletion in human erythrocytes in vitro (Calabrese et al., 1988; Evelo and Henderson, 1988; Fazi et al., 1991) and in vivo (Evelo and Henderson, 1992; Mulders, 1995).

Erythrocytes also contain mixed-function oxidase (MFO) activity capable of generating GSH-reactive compounds, an activity that according to Belvedere and Tursi (1981) must be attributed to oxyhaemoglobin. Erythrocytes are, for instance, known to metabolize styrene into styrene oxide (Belvedere and Tursi, 1981; Norppa et al., 1983; Tursi et al., 1983), benzo[ $a]$ pyrene into quinones (Lo Jacono et al., 1992) and heterocyclic amines into intermediates with mutagenic activity (Duverger-van Bogaert et al., 1991), to hydroxylate aniline (Mieyal et al., 1976), 
and to perform oxidative demethylation on methylaniline with concomitant release of formaldehyde (Stecca and Duverger-van Bogacrt, 1989).

For most compounds, the activity of electrophilic metabolite formation is much higher in the liver than in erythrocytes (Guengerich and Liebler, 1985). Although liver-generated electrophilic metabolites may be detoxified within the hepatocytes, they can also be excreted, enter the erythrocytes in the sinusoids and deplete erythrocyte glutathione.

In the present investigation, attention was paid to the interplay between the bioactivation and inactivation functions of liver and blood. The main questions, approached in vitro, were as follows:

1. What is the importance of the bioactivating and inactivating potency of red blood cells in comparison with the hepatic capacity?

2. Are erythrocytes accessible for GSH-reactive electrophilic compounds?

3. Is the outer cell membrane of hepatocytes a barrier to reactive metabolites formed intracellularly, or is an effective detoxification occurring intrahepatically?

We developed an incubation system using different combinations of human erythrocytes, haemolysate and aqueous GSH solution with rat liver microsomes or intact hepatocytes to mimic the in vivo situation. The choice of two different species for target cells (human erythrocytes) and metabolizing

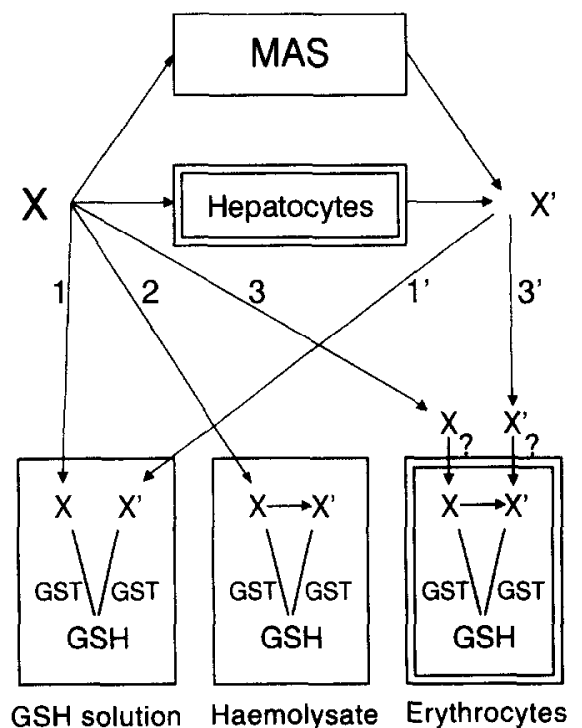

Fig. 1. An outline of the incubations performed in this study. GSH-reactive compounds (X) can react with GSH in aqueous solution or in haemolysates; if they are able to enter the erythrocyte they can also have this effect in this cell type. Non GSH-reactive compounds can sometimes be metabolized into reactive compounds $\left(\mathbf{X}^{\prime}\right)$. This can occur either in the erythrocyte itself, provided that the parent compound can enter the cell (if this is not the case effects may be seen only in haemolysates), or by added metabolic systems such as MAS or hepatocytes. The diagram is further explained in the text. cells (rat hepatocytes) was made to obtain an optimal combination of significance (human target, no substances with known qualitative differences in metabolism across species used) and feasibility (rodent liver, no large volumes of rodent blood). The test systems can easily be adapted to use human liver-derived activating systems, or blood of larger animals that may sometimes be more readily available.

An outline of the various experimental conditions studied in the present investigation is shown in Fig. 1. Direct reactivity of the substrate (X) with GSH was tested in incubations with aqueous GSH solutions (arrow 1, Fig. 1). Metabolic activity of erythrocytes for the test substrates (MFO plus GST) was tested through comparison of incubations with aqueous GSH solution and incubations with haemolysate (arrow 2). The influence of erythrocyte integrity on uptake and metabolism of the test compounds was tested by comparison of incubations with haemolysate and erythrocytes (arrow 3). This influence may consist of effects of the membrane on uptake and, for example, of differences in reductive capacity due to higher oxygen exposure of the haemolysates.

Biotransformation of the test compound by means of a microsomal activating system (MAS) into glutathione-reactive metabolites was tested through incubations with aqueous GSH solution with (arrow 1') and without MAS (arrow 1). These incubations were compared with incubations in which erythrocytes and MAS were combined (arrow $3^{\prime}$ ).

Biotransformation of the test compound by hepatocytes (both phase I and phase II activity) was assessed by determination of glutathione depletion. The ability of GSH-reactive metabolites to pass the hepatocyte membrane was also tested. Therefore, after incubation of substrate with hepatocytes, the intact cclls were removed by centrifugation and the supernatant was incubated again with GSH (arrow 1'). Co-incubations of hepatocytes and erythrocytes (arrow 3') were used to test whether metabolites generated and excreted by hepatocytes are able to enter the erythrocyte and deplete erythrocyte glutathione.

\section{MATERIALS AND METHODS}

\section{Chemicals}

Reduced glutathione (GSH), glucose-6-phosphate dehydrogenase (G6PDH; EC 1.1.1.49), glutathione reductase (EC 1.6.4.2), $\beta$-glucuronidase/arylsulfatase (EC 3.2.1.31/EC 3.1.6.1), glucose-6-phosphate (G6P), NADP $(H), N A D H$, cyclophosphamide (CP; CAS no. 6055-19-2) and 3-hydroxyacetanilide (3-HAA; CAS no. 621-42-1) were obtained from Sigma (St Louis, MO, USA). Diethyl maleate (DEM), $N$ ethylmaleimide (NEM) and iodoacetamide (IAcA) were from Janssen Chimica (Beerse, Belgium). 2Methylfurane (2-MF; CAS no. 534-22-5) was from Merck (Darmstadt, Germany), and monuron (MN; 
CAS no. 150-68-5) and monulinuron (MLN; CAS no. 1746-81-2) were from Riedel-de Haën (Seelse, Germany). Collagenase B (from Clostridium histolyticum) was obtained from Boehringer (Mannheim, Germany). Water was demineralized and microfiltered before use. All other chemicals were of analytical grade.

\section{Animals}

Male Brown Norway rats (BN/M) of about $250 \mathrm{~g}$ were used. Housing was as previously described (Palmen and Evelo, 1993). For phenobarbital pretreatment the animals received $1 \mathrm{~g} /$ litre of the substance in their drinking water for $1 \mathrm{wk}$ prior to the preparation of microsomes (PB microsomes).

\section{Biological preparations}

The preparation of the microsomal activating system was as described previously (Palmen and Evelo, 1993). The procedure for the isolation of hepatocytes was based on the methods described by Berry and Friend (1969) and by Seglen (1973). Viability of the cells (more than 90\%) was determined with the trypan blue exclusion test (Jauregui et al., 1981). All incubations using hepatocytes (both with and without erythrocytes) were performed in Krebs-Henseleit buffer of $\mathrm{pH}$ 7.4. Erythrocytes and haemolysates were prepared as previously described (Palmen and Evelo, 1993).

\section{Incubations}

The chemicals tested can be divided into direct GSH-reactive compounds (IAcA, NEM, DEM) and compounds that need further metabolization into GSH-reactive metabolites (CP, 3-HAA, 2-MF) (Garle and Fry, 1989). Two pesticides (MN and MLN) were also tested because their metabolite (4-chloroaniline) is known to generate haemoglobin adducts (Sabbioni and Neumann, 1990). As the glutathione sulfhydryl group is more sensitive to alkylation than the haemoglobin sulfhydryl group (Evelo and Henderson, 1988), MN and MLN might also affect glutathione concentrations.

Direct GSH-reactive compounds. Whole blood $(1.5 \mathrm{ml})$ or an equal volume of aqueous GSH solution in phosphate buffered saline (PBS, pH 7.3), were incubated in a shaking water-bath $\left(37^{\circ} \mathrm{C}, 210 \mathrm{rpm}\right)$ with $50 \mu 1$ of the test compounds IACA, DEM and NEM. Final concentrations were $0,0.5,1.0$ or $2 \mathrm{mM}$. IAcA and NEM were dissolved in PBS, and DEM in $100 \%$ ethanol. Addition of ethanol alone had no effect on the GSH values. The remaining GSH concentrations were determined after an incubation period of $1 \mathrm{hr}$ for GSH solutions (initial concentration $500 \mu \mathrm{M}$ ) and of $15 \mathrm{~min}$ for human blood (initial GSH concentrations 860,820 and $720 \mu \mathrm{M}$, for IAcA, DEM and NEM, respectively). All these experiments were performed in triplicate $(1 \times 3)$.

Other compounds. $2 \mathrm{ml}$ aqueous GSH solution, haemolysate or erythrocytes (final $\mathrm{Hb}$ concentrations 57 and $23 \mathrm{~g}$ /litre respectively) in $100 \mathrm{mM} \mathrm{Na} \mathrm{NPO}_{4} /$ $\mathrm{KH}_{2} \mathrm{PO}_{4}$ buffer (pH 7.4) were incubated at $37^{\circ} \mathrm{C}$ in a shaking water-bath $(80 \mathrm{rpm})$. After $5 \mathrm{~min}$ of preincubation, $1 \mathrm{ml}$ of the test compound (CP, 3-HAA, 2-MF, MN or MLN) was added. Final concentrations were between 0 and $2 \mathrm{~mm}$. Aqueous GSH and erythrocyte incubations were also performed in the presence of normal and PB-pretreated MAS, which was added before preincubation to a final protein concentration of $1 \mathrm{mg} / \mathrm{ml}$. After $1 \mathrm{hr}$ of incubation the samples were treated as previously described (Palmen and Evelo, 1993) and total glutathione (GT: the total of reduced plus oxidized glutathione) concentrations were measured. Initial GT concentrations were $440 \pm 5 \mu \mathrm{M}$ (mean \pm SE) for aqueous GSH solutions $(300 \pm 5 \mu \mathrm{M}$ for 2 -MF), $235 \pm 20 \mu \mathrm{M}$ for haemolysates and $110 \pm 4 \mu \mathrm{M}$ for erythrocytes. All experiments were performed three times and in triplicate $(3 \times 3)$. For CP and 3-HAA, separate experiments were performed for test compound concentrations between 0 and $0.5 \mathrm{~mm}$ and between 0.5 and $2.0 \mathrm{~mm}$.

Incubations with hepatocytes: Hepatocytes $(4.4 \times$ $10^{6} \mathrm{cells} / \mathrm{ml}$ ) plus $3-\mathrm{HAA}$ or CP solutions (final concentrations between 0 and $2.0 \mathrm{mM}$ ) were incubated in Krebs-Henseleit buffer (pH 7.4) for $1 \mathrm{hr}$ (incubation volume $1.5 \mathrm{ml}, 37^{\circ} \mathrm{C}$ ) in a shaking waterbath $(80 \mathrm{rpm})$. After the incubation period, $200 \mu \mathrm{l}$ trichloroacetic acid (TCA) $(8 \%)$ was added to an equal volume of hepatocyte suspension and GT was determined. The rest of the sample was centrifuged $(5 \mathrm{~min}, 25 \mathrm{~g}$ ) and $1 \mathrm{ml}$ supernatant plus $200 \mu \mathrm{l}$ GSH solution (final concentration between 100 and $400 \mu \mathrm{M})$ was incubated again under the same conditions. In order to liberate possible conjugated metabolites, $\beta$-glucuronidase/arylsulfatase was added (final activity of $650 \mathrm{U} / \mathrm{ml}$, with the $\mathrm{pH}$ adjusted to 6.2 by addition of $175 \mu 13 \mathrm{M} \mathrm{KH}_{2} \mathrm{PO}_{4}$ ). DEM pretreatment was performed by incubation of $4.4 \times$ $10^{6} \mathrm{cells} / \mathrm{ml}$ with $1 \mathrm{mM}$ DEM in Krebs-Henseleit buffer for $15 \mathrm{~min}$ in a shaking water-bath at $37^{\circ} \mathrm{C}$. Excess DEM was removed by washing twice with the same buffer.

Co-incubations of erythrocytes and hepatocytes: Coincubations of erythrocytes and hepatocytes were performed in transwells ${ }^{\mathrm{TM}}$ (Costar, Cambridge, UK), that are composed of a well in which a smaller well (transwell) can be placed containing a porous membrane $(0.4 \mu \mathrm{m})$. Erythrocytes $(2.6 \mathrm{ml}$, Hb concentration $27 \mathrm{~g} / \mathrm{litre}$ ) were pipetted into the well, and the hepatocytes $\left(1 \mathrm{ml}, 6.6 \times 10^{6}\right.$ cells $\left./ \mathrm{ml}\right)$ plus 3 -HAA $(0.5 \mathrm{ml}$, final concentration between 0 and $5 \mathrm{mM}$ ) were added to the transwell. After an incubation period of $1 \mathrm{hr}$, the erythrocytes were pipetted into plastic vials and packed by centrifugation. Further treatment was as previously described (Palmen and Evelo, 1993) and GT concentrations were determined. These 
experiments were performed three times in triplicate $(3 \times 3)$.

\section{Analytical procedures}

GSH was determined as previously described (Evelo and Henderson, 1988). GT in the TCA supernatants was determined after dilution (nine-fold) with buffer by the cyclic oxidation reduction method described by Anderson (1985). Lactate dehydrogenase (LDH) activity was determined using NADH consumption during pyruvate transformation to lactate (Moss et al, 1986). Microsomal and hepatocyte protein contents were determined according to Lowry et al. (1951). Haemoglobin concentrations were determined by measuring cyanmethaemoglobin according to van Kampen and Zijlstra (1965).

\section{Statistical analysis}

GT depletion caused by CP, 3-HAA, 2-MF, MN and MLN was analysed separately for both incubations with normal and PB microsomes by linear regression, using a model including control value and concentration as explanatory variables. Using that model we tested, for each metabolizing system, whether slopes of GT depletion versus concentration were significant.

\section{RESULTS}

Incubations without addition of metabolic activating system

Depletion of GSH by IACA, NEM and DEM and depletion of GT by CP, 3-HAA and 2-MF in incubations without MAS, is shown in Figs 2, 3 and 4 for aqueous GSH solutions, erythrocytes and haemolysates, respectively.

GSH in aqueous solution was depleted by the direct GSH-reactive compounds IAcA, NEM and DEM. The most reactive compound was NEM, which brought about almost complete depletion at

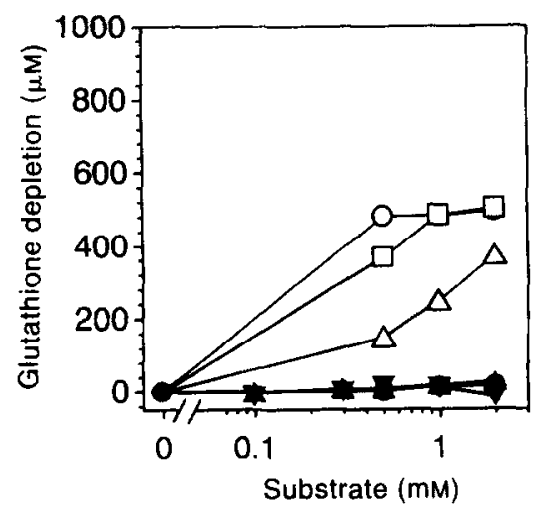

Fig. 2. GSH depletions in aqueous solution after incubation with the reactive compounds IAcA ( $\square)$, NEM $(O)$ and DEM $(\triangle)$ (single experiments in triplicate: $1 \times 3$ ) and GT depletion in incubations with $C P(\Delta), 3-H A A(\nabla)$ and 2-MF (O) (three experiments in triplicate: $3 \times 3$ ).

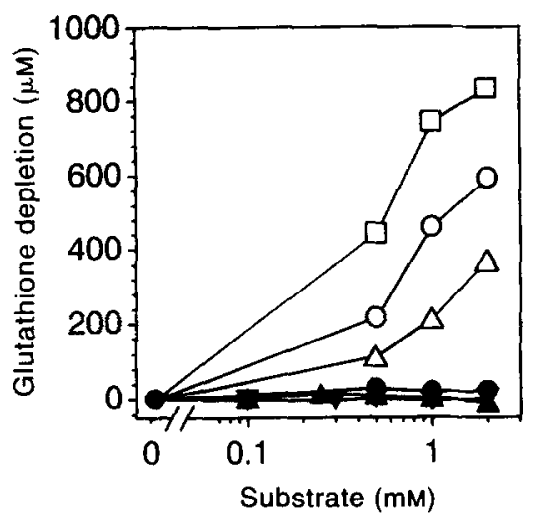

Fig. 3. GSH depletion in erythrocytes after incubation with IAcA $(\square)$, NEM $(O)$ and DEM $(\triangle)$ (single experiments in triplicate: $1 \times 3$ ) and $G T$ depletion in incubations with $\mathrm{CP}(\Delta), 3-\mathrm{HAA}(\boldsymbol{\nabla})$ and 2-MF (O) (three experiments in triplicate: $3 \times 3$ ).

$0.5 \mathrm{mM}$; for IAcA a comparable effect was reached at $1.0 \mathrm{mM}$. DEM was the least reactive compound. These three compounds also caused GSH depletion in erythrocytes, indicating that they do pass through erythrocyte membrane. Here, IAcA was the most effective compound, as $97 \%$ of the initial GSH concentration was depleted at a IAcA concentration of $2 \mathrm{mM}$, followed by NEM $(82 \%)$ and DEM $(45 \%)$.

Consistent with the fact that CP, 3-HAA, 2-MF, MN and MLN need metabolic activation, GT was not depleted in incubations of these compounds with aqueous GSH solution or erythrocytes. In incubations of haemolysates, concentration-dependent GT depletion $(P \leqslant 0.01)$ was found for 3-HAA (depletion of $18 \mu \mathrm{mol} / \mathrm{mmol} \mathrm{3-HAA}$ ) and 2-MF (12 $\mu \mathrm{mol} / \mathrm{mmol})$.

\section{Incubations with MAS}

In incubations with aqueous GSH solution and normal microsomes (Fig. 5), GT was depleted in a

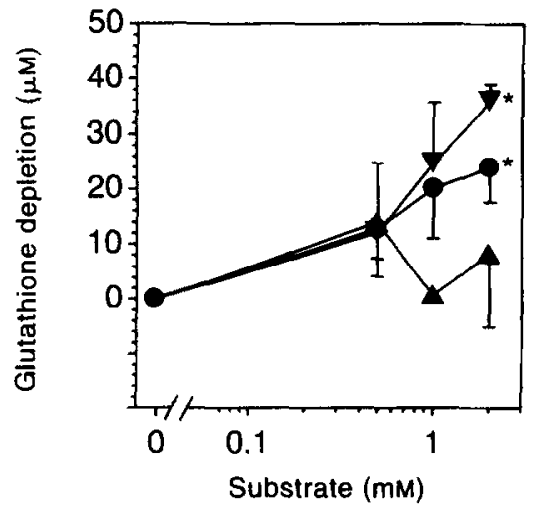

Fig. 4. GT depletion in haemolysates by $\mathrm{CP}(\mathbf{\Delta}), 3-\mathrm{HAA}$ $(\boldsymbol{\nabla})$ and $2-\mathrm{MF}(\boldsymbol{O})$. Mean and interexperimental SE are shown for three experiments performed in triplicate $(3 \times 3)$. The GT depletion was concentration dependent for 3-HAA and 2-MF: asterisks indicate significant differences from control $(* P \leqslant 0.01$ in both cases; linear regression). 


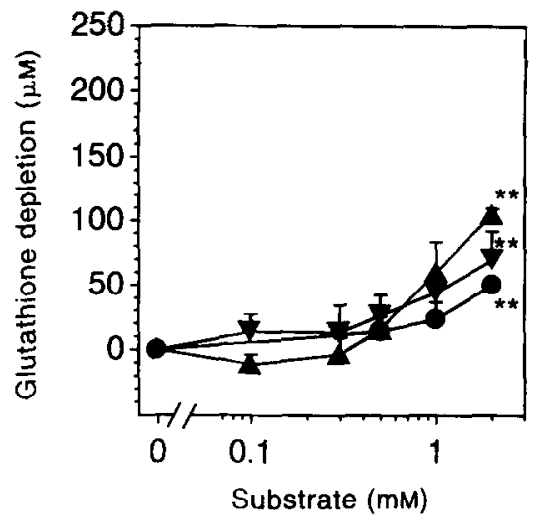

Fig. 5. GT depletion in incubations $(3 \times 3)$ of aqueous GSH solution and microsomes of untreated rats with $C P(A)$, 3-HAA ( $\nabla$ ) and 2-MF (O). The mean and the interexperimental SE are shown. The GT depletion was concentration dependent for all three compounds: asterisks indicate significant differences from control $(* * P \leqslant 0.001$ in all cases; linear regression).

concentration-dependent way by $\mathrm{CP}$ (GT depletion was $48 \mu \mathrm{mol} / \mathrm{mmol}), 3-\mathrm{HAA}(36 \mu \mathrm{mol} / \mathrm{mmol})$ and 2-MF ( $25 \mu \mathrm{mol} / \mathrm{mmol})(P \leqslant 0.001 \mathrm{in}$ all three cases), and not by $\mathrm{MN}$ or MLN. When PB microsomes were used (Fig. 6) even higher depletions were found for CP $(92 \mu \mathrm{mol} / \mathrm{mmol}), 2-\mathrm{MF}(41 \mu \mathrm{mol} / \mathrm{mmol})$ and 3-HAA. For the latter compound a plateau in GT depletion was found at 3-HAA concentrations above $0.3 \mathrm{~mm}$ where the initial GSH concentration was about $25 \%$ depleted. For these microsomes, also, MN and MLN had no effect.

The same profile was found for erythrocytes. In the presence of normal microsomes (Fig. 7), GT was depleted in a concentration-dependent way by $\mathrm{CP}$ (46 $\mu \mathrm{mol} / \mathrm{mmol} \mathrm{CP}), 3-\mathrm{HAA}(11 \mu \mathrm{mol} / \mathrm{mmol})$ and $2-\mathrm{MF}(23 \mu \mathrm{mol} / \mathrm{mmol})(P \leqslant 0.001$ in all cases $)$. In the

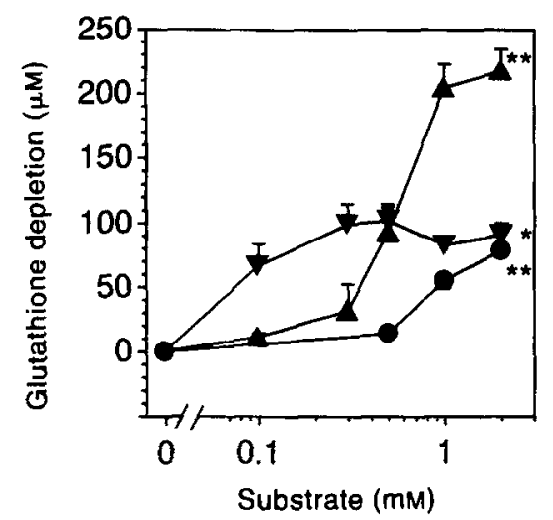

Fig. 6. GT depletion in incubations $(3 \times 3)$ of aqueous GSH solution and microsomes of PB-pretreated rats with $\mathrm{CP}(\boldsymbol{A})$, 3-HAA ( $\boldsymbol{\nabla})$ and 2-MF (O). The mean and the interexperimental SE are shown. In some cases the SE falls within the datapoints. The GT depletion was concentration dependent for all three compounds: asterisks indicate significant differences from control $\left({ }^{*} P \leqslant 0.01\right.$ for 3 -HAA;

${ }^{* *} P<0.001$ for $\mathrm{CP}$ and $2-\mathrm{MF}$; linear regression).

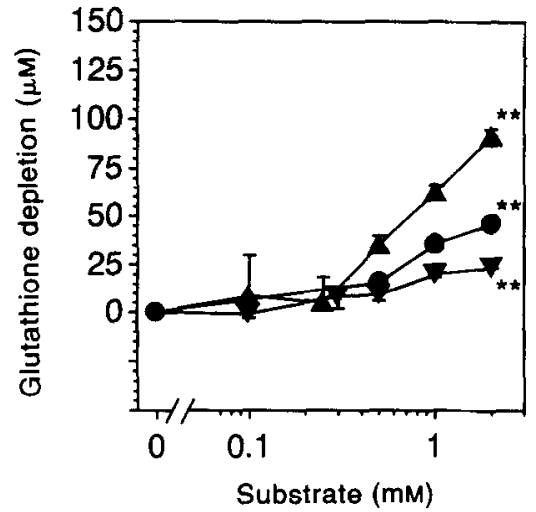

Fig. 7. GT depletion in incubations $(3 \times 3)$ of erythrocytes and microsomes of untreated rats with $\mathrm{CP}(\Delta), 3-\mathrm{HAA}(\boldsymbol{\nabla})$ and 2-MF (O). The mean and interexperimental $\mathrm{SE}$ are shown. In some cases the SE falls within the datapoints. The GT depletion was concentration dependent for all three compounds: asterisks indicate significant differences from control (**P $\leqslant 0.001$; linear regression).

presence of PB microsomes (Fig. 8), even more marked depletion was found for CP (leading to full depletion at $0.5 \mathrm{mM} \mathrm{CP}$ ), 3-HAA (depletion of $30 \mu \mathrm{mol} / \mathrm{mmol} 3-\mathrm{HAA})$ and $2-\mathrm{MF}(39 \mu \mathrm{mol} / \mathrm{mmol})$. Again MN and MLN had no effect, irrespective of the kind of microsomes used.

All effects of microsomes reported above were clearly dependent on the presence of an NADPHregenerating system.

\section{Incubations with hepatocytes}

Viability of hepatocytes. The viability of hepatocytes after incubation was tested by measurement of LDH leakage into the medium (Jauregui et al., 1981). LDH leakage was low during the first $50 \mathrm{~min}$ of the incubation period and increased thereafter (data not

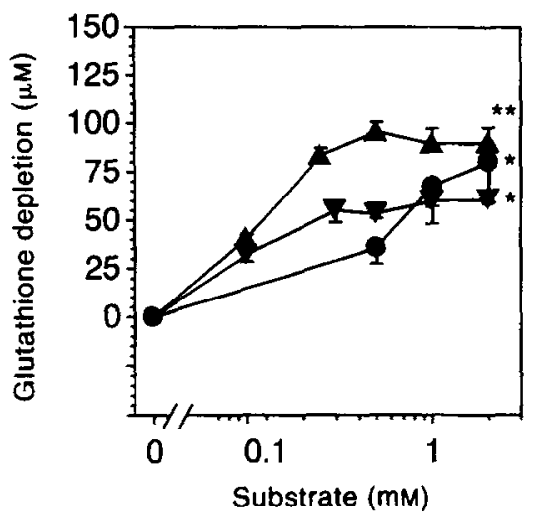

Fig. 8. GT depletion in incubations $(3 \times 3)$ of erythrocytes and microsomes of PB-pretreated rats with CP $(\Delta), 3-$ HAA $(\nabla)$ and 2-MF (O). The mean and interexperimental SE are shown. The GT depletion was concentration dependent for all three compounds: asterisks indicate significant differences from control $\left({ }^{*} P \leqslant 0.01\right.$ for $3-\mathrm{HAA}$ and $2-\mathrm{MF}$; ${ }^{* *} P \leqslant 0.001$ for $C P$; linear regression). 
Table 1. Glutathione depletion and excretion of GSH-reactive compounds from hepatocytes incubated with 3-HAA and CP

\begin{tabular}{|c|c|c|c|}
\hline Compound & $\begin{array}{c}\text { Concentration } \\
(\mathrm{mM})\end{array}$ & $\begin{array}{c}\text { GT depletion } \\
\text { ( } \mu \text { mol } / \mathrm{g} \text { protein })\end{array}$ & $\begin{array}{c}\text { Excretion } \\
(\mu \mathrm{M})\end{array}$ \\
\hline \multirow[t]{2}{*}{ 3-HAA } & 1.0 & $6.0 \pm 1.7(n=3)$ & $0.6(n=1)$ \\
\hline & 2.0 & $9.5 \pm 1.4(n=5)$ & $10.4(n=2: 6.7,14)$ \\
\hline \multirow[t]{2}{*}{$C \cdot P$} & 1.0 & $15.1(n=1)$ & n.d. \\
\hline & 2.0 & $15.0(n=2: 10.2,19.8)$ & $6(n=1)$ \\
\hline \multicolumn{4}{|c|}{$\begin{array}{l}\text { All values were determined after } 1 \mathrm{hr} \text { incubation of hepatocytes with } 3 \text {-HAA or CP. Where } \\
\text { applicable, the mean } \pm \text { standard error of the depletion/g protein }(4 \mathrm{~g} / \text { litre in the incubations) } \\
\text { is given for the number of experiments shown; each experiment consisted of incubations in } \\
\text { triplicate. Excretion of GSH reactive products was determined by reincubation }\left(1 \mathrm{hr}, 37^{\circ} \mathrm{C}\right) \\
\text { with GSH. }\end{array}$} \\
\hline
\end{tabular}

shown). The LDH leakage after $\mathrm{I} \mathrm{hr}$ of incubation was not increased by addition of 3-HAA or CP at concentrations up to $2 \mathrm{mM}$. Incubation of hepatocytes with DEM (final concentration $1 \mathrm{~mm}, 15 \mathrm{~min}, 37^{\circ} \mathrm{C}$ ) depleted $75 \pm 5 \%$ of the initial GT concentration but had no effect on the LDH leakage.

Incubations of hepatocytes alone. The effects of 3-HAA and CP on hepatocyte GT and the excretion of GSH-reactive compounds are shown in Table 1. A concentration-dependent hepatocyte GT depletion was found for 3-HAA $(P \leqslant 0.01)$. At 2 mM 3-HAA, this depletion amounted to about $10 \mu \mathrm{mol} / \mathrm{g}$ protein. In two experiments with $\mathrm{CP}$, comparable or somewhat higher depletions (10 and $20 \mu \mathrm{mol} / \mathrm{g}$ protein at $2 \mathrm{~mm}$ ) were found.

The ability of GSH-reactive metabolites to pass the hepatocyte membrane was tested. After completion of the incubation with test compound, hepatocytes were removed by centrifugation and the supernatants were reincubated with GSH for $1 \mathrm{hr}$ under the same conditions. Table 1 shows that the GT concentration in the supernatants decreased as a result of the earlier incubation of hepatocytes with the test compound, indicating that GSH-reactive 3-HAA and CP metabolites were excreted by the hepatocytes. In one experiment part of the hepatocytes were pretreated with DEM, leading to $75 \pm 5 \%$ GT depletion in the hepatocytes. After this pretreatment the excretion of GSH-reactive 3-HAA metabolites increased from 0.6 to $6.6 \mu \mathrm{M}$ at $1 \mathrm{mM} 3-\mathrm{HAA}$ and from 6.7 to $17.8 \mu \mathrm{M}$ at 2 mM 3-HAA. Electrophiles generated by hepatocytes may also be detoxified by conjugation to compounds other than GSH and excreted into the medium. Rashed et al. (1990) showed that $80 \%$ of 3-HAA metabolites formed in mice are sulfated or glucuronidated. We tried to liberate GSH-reactive compounds from such metabolites by adding $\beta$ glucuronidase/arylsulfatase to the medium of the second (GSH containing) incubation. However, addition of the esterases did not increase the GT depletion.

Co-incubations of erythrocytes and hepatocytes. Erythrocyte GT concentration was not affected when erythrocytes and hepatocytes were co-incubated. Addition of 3-HAA to these incubations (final concentrations up to $5 \mathrm{~mm}$ ) did not affect erythrocyte $\mathrm{GT}$, neither when normal hepatocytes were used nor when hepatocytes from PB-pretreated rats were tested. In an attempt to increase the amount of GSH-reactive 3-HAA metabolites, the hepatocytes were pretreated with DEM. Although this led to $72 \%$ depletion in the hepatocytes, it did not affect the erythrocyte GT concentration. Similarly, isolation and incubation of 3-HAA-treated hepatocytes in sulfate-free medium-known to affect sulfation capacity (Evelo et al., 1984)-had no effect on erythrocyte GT in co-incubations.

\section{DISCUSSION}

Most cell types are more or less able to (de)toxify foreign chemicals. The hepatocyte is known to be a metabolically very active cell and erythrocytes have some metabolic activity also (Belvedere and Tursi, 1981). Foreign compounds, toxified by hepatocytes by means of cytochrome $P-450$ activity, may be detoxified by phase II metabolism (e.g. glucuronidation, sulfation, glutathione conjugation). This may lead to a shortage of hepatocyte phase II conjugation factors such as UDP-glucuronic acid, 3'-phosphoadenosine-5'phosphosulfate and GSH. Conjugated and/or unconjugated metabolites may be released by the hepatocyte into the sinusoids; this means that erythrocytes can be exposed to these metabolites. When the metabolites are able to pass through the erythrocyte membrane, the biochemical functions of the erythrocyte may be affected.

In the present investigation, we developed a set of in vitro test systems (Fig. 1) that may help to shed light on the interplay between the bioactivation and inactivation functions of liver and blood, using glutathione depletion as the dependent variable. A comparable method was described by Tingle and Park (1993), who noted methaemoglobin formation in human erythrocytes incubated with dapsone in the presence of rat liver microsomes. To approach the in vivo situation more closely, we tested whether hepatocytes are able to generate GSH-reactive metabolites from CP or 3-HAA, and the ability of 3-HAA metabolites to deplete erythrocyte GT. As a large variety of variables (besides glutathione depletion) can be tested, the test systems may extend the current set of techniques. GSH is an efficient scavenger of reactive electrophilic substances, especially 
in combination with glutathione $S$-transferases (DeLeve and Kaplowitz, 1991; Mannervik and Danielson, 1988; Meister and Anderson, 1983). Because of this property, a decrease of the GSH concentration (glutathione depletion), caused by the test compound, has been used as a measure of the amount of GSH-reactive compounds in the incubate (Garle and Fry, 1989 and 1990; Garle et al., 1988).

IACA, NEM and DEM are direct GSH-reactive compounds that need no metabolic activation (Evelo and Henderson, 1988). In incubations with aqueous GSH solution and in incubations with erythrocytes, GSH was, indeed, depleted. The depletion of GSH in solutions without biological material not only confirms that these compounds need no metabolic activation but also shows that the reactions are not dependent on GST. The depletion in erythrocytes proves that the compounds are able to pass through the erythrocyte membrane. CP, 3-HAA, 2-MF, MN and MLN did not influence GT concentrations (GT is the sum of both reduced and oxidized glutathione) of aqueous GSH solutions or erythrocytes when no external metabolizing system was added, indicating that these compounds have no direct reactivity with GSH.

Both 3-HAA and 2-MF depleted GT in haemolysate but not in incubations with aqueous GSH solution or erythrocytes. The results indicate that a factor in the haemolysate is capable of metabolizing 3-HAA and 2-MF, resulting in GSH conjugation. This metabolic activity cannot be explained by mere GST-catalysed glutathione conjugation to the parent compounds, as the addition of glutathione $S$ transferase $\pi(3.2 \mathrm{U} / \mathrm{ml})$ to the incubations with aqueous GSH solution and 3-HAA or 2-MF, had no effect on the GT concentration (data not shown).

Only when rat MAS was added to incubations of CP, 3-HAA or 2-MF and aqueous GSH solution or erythrocytes, was GT depleted. GT depletions were concentration dependent when normal or PB microsomes were used. The presence of NADPH was a requirement. This indicates that metabolites of $\mathrm{CP}$, 3-HAA and 2-MF are generated by MAS and that these metabolites are able to enter the erythrocyte. For all three compounds tested, GT depletions were higher in incubations with aqueous GSH solution than in erythrocytes when normal microsomes were tested. This may indicate that the erythrocyte membrane acts as a barrier for metabolites of CP, 3-HAA and $2-\mathrm{MF}$, generated by microsomes. If the erythrocyte membrane would limit diffusion to less than $80 \mathrm{nmol} / \mathrm{hr} / \mathrm{m}^{2}$ membrane, GT depletion would be less than $10 \%$ during the assay period and therefore probably would not be detectable. [The incubated amount of erythrocytes corresponds to $200 \mu \mathrm{l}$ cells, containing $300 \mathrm{nmol}$ GSH. The amount of GSHreactive metabolite needed to deplete $10 \%$ of this GSH pool would be $30 \mathrm{nmol}$. The membrane surface of $200 \mu 1$ erythrocytes is $0.36 \mathrm{~m}^{2}$ (Guyton, 1986)].
MAS-dependent depletions by CP, 3-HAA and 2-MF were inducible by PB.

Despite the fact that MN and MLN are known to give rise to (4-chloroanilin) $\mathrm{Hb}$ adducts in rats (Sabbioni and Neumann, 1990), and were therefore expected to have some activity towards GSH after metabolization, we did not find any such effect.

Hepatocyte GT decreased by about $10 \mu \mathrm{mol} / \mathrm{g}$ protein in incubations with $2 \mathrm{~mm} 3-\mathrm{HAA}$, and by at least this amount with $2 \mathrm{mM} \mathrm{CP}$. Since the protein concentration of hepatocytes is about $200 \mathrm{~g} / \mathrm{litrc}$, this corresponds to a GT depletion in the hepatocytes of about $2 \mathrm{~mm}$. To verify whether substantial fractions of the metabolites formed were excreted by the hepatocytes, the supernatants of the 3-HAA or CP hepatocyte incubations were reincubated with GSH during $1 \mathrm{hr}$ under the same conditions. The GT depletion in the supernatant of incubations with 3-HAA ( $2 \mathrm{mM}$ ) was of the order of $10 \mu \mathrm{M}$. Since the mean GT depletion in the hepatocytes corresponded to $45 \mathrm{nmol} / \mathrm{ml}$ incubate, this means that the excreted fraction was about $20 \%$. It should be noted that the method used for the measurement of excreted metabolites detects only the more stable fraction of these metabolites that are still present after $1 \mathrm{hr}$ of incubation. Because of this it is likely that the actual amount of such excreted metabolites was (much) higher. The calculated excreted fraction must therefore be considered as the lower limit of the actual excreted fraction. Since the intracellular GSH depletions for $\mathrm{CP}$ were at least as high as those for 3-HAA, and since the excretion seemed to be somewhat lower for $\mathrm{CP}$, the excreted fraction of $\mathrm{CP}$ metabolites is probably also lower. This led us to the use of 3-HAA in the co-incubations of hepatocytes and erythrocytes. For technical reasons enforced by the geometry of the 'transwells', the dilution of excreted metabolites was even higher in these coincubations (incubation volume $4.1 \mathrm{ml}$ instead of $1.5 \mathrm{ml}$ ), leading to a calculated 3-HAA metabolite concentration of $3.7 \mu \mathrm{M}$ outside the erythrocytes at the end of the incubation. Even if we suppose that all of this substance enters the erythrocytes during the experiment, erythrocyte GT depletion would have been only $5 \%$ and therefore not detectable. As explained above, the actual metabolite concentration during the experiments may have been higher than that detected at the end of the hepatocyte incubations. If we assume, for instance, that the actual metabolite concentration was about $40 \mu \mathrm{M}$ (i.e. 10 times higher than that actually detected), the necessary permeability coefiicient $(\mathrm{P})$ for unidirectional diffusion can be calculated from $\mathrm{P}=[\mathrm{V} /(\mathrm{A} \times \Delta \mathrm{c})] \mathrm{dc} / \mathrm{dt}$ (Rubinow, 1975). Where the volume of erythrocytes in the incubation (V) is $200 \mu \mathrm{l}$, the surface area of those erythrocytes (A) is $0.36 \mathrm{~m}^{2}$ (see above), the calculated concentration gradient over the cell membranes is $40 \mu \mathrm{M}$ and the change of the intracellular erythrocyte concentration $(\mathrm{dc} / \mathrm{dt})$ must be at least $10 \%$ of the GSH concentration $(10 \%$ of $1.5 \mathrm{~mm})$ in $1 \mathrm{hr}$. This 
means that $P$ must be at least $5.8 \times 10^{-8} \mathrm{~cm} / \mathrm{sec}$. For comparison, known values are about $10^{-7} \mathrm{~cm} / \mathrm{sec}$ for glucose and $10^{-10} \mathrm{~cm} / \mathrm{sec}$ and lower for ions (Alberts et al., 1983). This indicates that even with this 10 -fold higher excretion, effective depletion would have been detectable within $1 \mathrm{hr}$ only when the diffusion uptake speed of the metabolites was at least $60 \%$ of the uptake speed of glucose. The two most important factors limiting erythrocyte GSH depletion are therefore (a) the total erythrocyte concentration (higher amounts of erythrocytes leading to higher amounts of GSH that are more difficult to deplete in substantial amounts) and (b) total incubation volume (higher volumes leading to dilution of metabolites and thereby to a lower metabolite gradient over the membrane and thus to lower diffusion speed).

To increase the excretion of GSH-reactive metabolites by the hepatocytes, hepatocytes were preincubated with diethyl maleate in order to deplete their glutathione store. Whereas this treatment led to over $70 \%$ depletion of GT in the hepatocytes, no effect of this treatment on erythrocyte GT was found. Since sulfation by isolated hepatocytes can be reduced extensively when no inorganic sulfate is available (Evelo et al., 1984), this treatment may increase the amount of unconjugated reactive metabolites in the hepatocytes and, in case of excretion, in the supernatant. However, isolation of hepatocytes with sulfate-free buffer solutions, and incubation of hepatocytes and erythrocytes in sulfate-free Krebs buffer, did not deplete erythrocyte GT when 3-HAA was tested. Addition of $\beta$-glucuronidase/arylsulfatase to incubations composed of GSH and supernatant that was previously incubated with hepatocytes and 3-HAA, also had no effect on GT depletions.

In summary, the direct GSH-reactive compounds IAcA, NEM and DEM were able to enter the erythrocyte and deplete the erythrocyte GSH store. CP, 3-HAA and 2-MF depleted erythrocyte GT only when MAS was added, indicating that GSH-reactive metabolites were formed that were able to enter the erythrocyte. Hepatocytes also metabolized 3-HAA and CP into GSH-reactive metabolites. They were able to excrete a fraction of these GSH-reactive metabolites into the supernatant. For 3-HAA, no erythrocyte GT depletion was found in coincubations of hepatocytes and erythrocytes, which may be explained by uptake limitation of the GSHreactive metabolites into the erythrocytes.

Acknowledgements - We wish to thank Professor Dr P. Th. Henderson for critical reading of the manuscript, Dr A. Kester for his advice on the statistical analyses and the Red Cross Blood Bank 'Zuid Limburg' for collecting the blood samples.

\section{REFERENCES}

Alberts B., Bray D., Lewis J., Raff M., Roberts K. and Watson J. D. (1983) Molecular Biology of the Cell.p. 287. Garland, New York.
Anderson M. E. (1985) Determination of glutathione and glutathione disulfide in biological samples. Methods in Enzymology 113, 548-555.

Belvedere G. and Tursi F. (1981) Styrene oxidation to styrene oxide in human blood erythrocytes and lymphocytes. Reseach Communications in Chemical Pathology and Pharmacology 33, 273-282.

Berry M. N. and Friend D. S. (1969) High yield preparation of isolated rat liver parenchymal cells. Journal of Cell Biology 43, 506-520.

Calabrese E. J., Tilli F., Horton H. M. and Stoddard A (1988) The effects of ethanol on the hematotoxicity of twelve pharmaceutical and environmental agents. Journal of Environmental Science and Health A23, 359-367.

DeLeve L. D. and Kaplowitz N. (1991) Glutathione metabolism and its role in hepatotoxicity. Pharmacology and Therapeutics 52, 287-305.

Duverger-van Bogaert M., Crutzen-Fayt M. C. and Stecca C. (1991) Mutagenicity of some heterocyclic amines in Salmonella typhimurium with metabolic activation by human red blood cell cytosol. Mutation Research 261, 261-265.

Evelo C. T. A. and Henderson P. Th. (1988) Influence of glutathione on the formation of cysteine alkylation products in human hemoglobin. Toxicology 52, 177-186.

Evelo C. T. A. and Henderson P. Th. (1992) Biological effect monitoring. Archives of Toxicology (Suppl. 15), 268-277.

Evelo C. T. A., Versteegh J. F. M. and Blaauboer B. J. (1984) Kinetics of the formation and secretion of the aniline metabolite 4-aminophenol and its conjugates by isolated rat hepatocytes. Xenobiotica 14, 409-416.

Fazi A., Mancini U., Piatti E., Accorsi A. and Magnani M. (1991) Human red blood cells as bioreactors for the inactivation of harmful xenobiotics. Biotechnology and Applied Biochemistry 14, 60-68.

Garle M. J. and Fry J. R. (1989) Detection of reactive metabolites in vitro. Toxicology 54, 101-110.

Garle M. J. and Fry J. R. (1990) Reactive metabolite formation catalysed by cytochrome P-450j. Toxicology in Vitro 4, 493-496.

Garle M. J., Khan J. and Fry J. R. (1988) Depletion of glutathione by the hepatotoxins paracetamol and bromobenzene, and their non-hepatotoxic analogues, in a fortified liver microsomal system. Toxicology in Vitro 2 , 247-252.

Guengerich F. P. and Liebler D. C. (1985) Enzymatic activation of chemicals to toxic metabolites. Critical Reviews in Toxicology 14, 259-307.

Guyton A. C. (1986) Textbook of Medical Physiology. 7th Ed. p. 42. W. B. Saunders, Philadelphia.

Jauregui H. O., Hayner N. T., Driscoll J. L., WilliamsHolland R., Lipsky M. H. and Galletti P. M. (1981) Trypan blue dye uptake and lactate dehydrogenase in adult rat hepatocytes - freshly isolated cells, cell suspensions, and primary monolayer cultures. In Vitro Cellular and Developmental Biology 17, 1100-1110.

Lo Jacono F., Stecca C. and Duverger M. (1992) Mutagenic activation of benzo[a]-pyrene by human red blood cells. Mutation Research 268, 21-26.

Lowry O. H., Rosebrough N. J., Farr A. L. and Randall R. J. (1951) Protein measurement with the Folin phenol reagent. Journal of Biological Chemistry 193, 265-275.

Mannervik B. and Danielson U. H. (1988) Glutathione transferases: structure and catalytic activity. Critical Reviews in Bivchemistry 23, 283-337.

Meister A. and Anderson M. E. (1983) Glutathione. Annual Review of Biochemistry 52, 711-760.

Mieyal J. J., Ackerman R. S., Blumer J. L. and Freeman L. S. (1976) Characterization of enzyme-like activity of human hemoglobin: properties of the haemoglobin-P-450 reductase-coupled aniline hydroxylase system. Journal of Biological Chemistry 251, 3436-3441. 
Moss D. W., Henderson A. R. and Kachmar J. F. (1986) Enzymes. In Textbook of Clinical Chemistry. Edited by N. W. Tietz. p. 1532. W. B. Saunders, Philadelphia.

Mulders T. M. T. (1995) In vivo characterization of glutathione conjugation in humans. PhD thesis, Leiden University.

Norppa H., Vainio H. and Sorsa M. (1983) Metabulic activation of styrene by erythrocytes detected as increased sister chromatid exchanges in cultured human lymphocytes. Cancer Research 43, 3579-3582.

Palmen N. G. M. and Evelo C. T. A. (1993) Glutathione depletion in human erythrocytes as an indicator for microsomal activation of cyclophosphamide and 3hydroxyacetanilide. Toxicology 84, 157-170.

Rashed M. S., Myers T. G. and Nelson S. D. (1990) Hepatic protein arylation, glutathione depletion, and metabolite profiles of actaminophen and a non-hepatotoxic regioisomer, 3'-hydroxyacetanilide, in the mouse. Drug Metabolism and Disposition 18, 765-770.

Rubinow S. I. (1975) Introduction to Mathematical Biology. Wiley \& Sons, New York.

Sabbioni G. and Neumann H. G. (1990) Biomonitoring of arylamines: hemoglobin adducts of urea and carbamate pesticides. Carcinogenesis 11, 111-115.

Seglen P. O. (1973) Preparation of rat liver cells. Experimental Cell Research 82, 391-398.

Stecca C. and Duverger-van Bogaert M. (1989) $N$ Demethylation reactions in intact erythrocytes and erythrocyte supernatant. Archives of Toxicology (Suppl. 13), 291-293.

Strange R. C., Johnson P. H., Lawton A., Moult J. A., Tector M. J., Tyminski R. J. and Cotton W. (1982) Studies on the variability of glulathione $S$-transferase from human erythrocytes. Clinica Chimica Acta 120, 251-260.

Tingle M. D. and Park B. K. (1993) The use of a three compartment in vitro model to investigate the role of hepatic metabolism in drug-induced blood dyscrasias. British Journal of Clinical Pharmaceutics 36, 31-38.

Tursi F., Samaia M., Salmona M. and Belvedere G. (1983) Styrene oxidation to styrene oxide in human erythrocytes is catalyzed by oxyhemoglobin. Experientia 39, 593-594. van Kampen E. J. and Zijlstra W. G. (1965) Determination of hemoglobin and its derivates. Advances in Clinical Chemistry 8, 141-187. 